\title{
Perioperative analgesia with a buprenorphine transdermal patch for hallux valgus surgery: a prospective, randomized, controlled study
}

This article was published in the following Dove Press journal: Journal of Pain Research

\author{
Can Xu \\ Mingqing Li \\ Chenggong Wang \\ Hui Li \\ Hua Liu \\ Department of Orthopaedics, Xiangya \\ Hospital, Central South University, \\ Changsha, Hunan Province, People's \\ Republic of China
}

Correspondence: Hua Liu Department of Orthopaedics, Xiangya Hospital, Central South University, No 87, Xiangya Road, Changsha 410008, Hunan Province, People's Republic of China

Tel +8615973138510

Fax +862184327224

Email liuhua345@I63.com
Purpose: Hallux valgus surgery often results in significant postoperative pain. Adequate control of pain is essential for patient satisfaction and improves the outcome of the procedure. This study aimed to investigate the perioperative analgesic effect of a buprenorphine transdermal patch in patients who underwent hallux valgus surgery.

Patients and methods: A total of 90 patients were randomly divided into the following three groups based on the perioperative analgesic method: flurbiprofen axetil intravenous injection (Group F), oral celecoxib (Group C), and buprenorphine transdermal delivery system (BTDS) (Group BTDS). The pain status, degree of satisfaction, adverse effects, and administration of tramadol hydrochloride for uncontrolled pain were recorded on the night before surgery, postoperative day 1, postoperative day 2, and postoperative day 3.

Results: The BTDS could effectively control perioperative pain for patients undergoing hallux valgus surgery. The analgesic effect of the BTDS was better than that of oral celecoxib. In addition, statistically significant differences were not observed in the visual analog scale (VAS) scores, adverse effects, and rescue analgesia between the patients who received the BTDS and the patients who received the flurbiprofen axetil intravenous injection. However, the degree of patient satisfaction of the BTDS group was significantly higher $(P<0.05)$ than that of the other two groups.

Conclusion: The BTDS (a preemptive analgesia regimen) could exert an analgesic effect during the perioperative period for patients who had received hallux valgus surgery, and this effect is beneficial for sustaining postoperative physiological and psychological states and promoting functional rehabilitation.

Keywords: hallux valgus, buprenorphine transdermal patch, analgesia, perioperative

\section{Introduction}

Hallux valgus surgery can be particularly painful in the immediate postoperative period. ${ }^{1,2}$ Many patients with hallux valgus express moderate-to-severe pain 2-3 days postoperatively, ${ }^{3}$ which seriously affects the postoperative physical and psychological status and functional rehabilitation of patients. If pain and nausea, the two major complaints after hallux valgus surgery, ${ }^{4}$ can be prevented or successfully resolved, then most of the patients scheduled for hallux valgus surgery will be suitable for day-case surgery.

Methods used to control pain include pharmacological analgesics, both oral and intravenous, and more invasive techniques, such as a continuous infusion of an anesthetic agent to maintain a nerve block during the immediate postoperative period. ${ }^{5}$ 
The buprenorphine transdermal delivery system (BTDS; Norspan ${ }^{\circledR}$; Mundipharma (China) Pharmaceutical Co., Ltd, Beijing, People's Republic of China) is a type of transdermal patch that contains an analgesic drug (buprenorphine in the case of the BTDS). The BTDS continuously delivers dosages of $5,7.5,10,15$, or $20 \mathrm{mg} / \mathrm{h}$ of buprenorphine steadily over 7 days, ${ }^{6,7}$ thereby administering a controlled release of the medication to patients with chronic pain. ${ }^{8-10}$ Improvements in the health-related quality of life of patients have been demonstrated in post hoc analyses of randomized clinical trials of patients with pain who were treated by the BTDS. ${ }^{11}$ However, few studies have been performed on the use of the BTDS for forefoot surgeries. Therefore, we designed a prospective, randomized, controlled study to evaluate the analgesic effectiveness and safety of the BTDS for hallux valgus surgery.

\section{Patients and methods}

\section{General data}

This was a randomized controlled study and was approved by the Severance Institutional Review Board of Xiangya Hospital at Central South University. The study protocol was designed in accordance with the Declaration of Helsinki guidelines, and all patients signed a written informed consent form. The study is also registered at chictr.org. Both the protocol and the statistical analysis plan are available at chictr.org.

Patients with mild and low-moderate hallux valgus who underwent hallux valgus correction under peripheral nerve blocks between October 2015 and July 2017 were enrolled in the study. All patients underwent a unilateral procedure. We use random number table method, and the patients were randomly divided into the following three groups based on the perioperative analgesic method used: flurbiprofen axetil intravenous injection (Group F), oral celecoxib (Group C), and BTDS (Group BTDS) ( $\mathrm{n}=30$ patients for each group). No significant differences in age, gender, height, weight, and body mass index (BMI) of the patients were found between the groups (Table 1). The preoperative visual analog scale (VAS) scores, mean preoperative hallux valgus angles and intermetatarsal angles, and total surgical times of the three groups were also similar (Table 2).

The inclusion criteria were as follows: 1) patients who underwent surgery for mild and low-moderate hallux valgus deformities; 2) patients between the ages of 18 and 75 years; 3) patients with American Society of Anesthesiologists grades I and II; 4) patients who underwent hallux valgus correction; and 5) patients who could understand the study procedures and assessment and agreed to participate in the study by giving written informed consent. The exclusion criteria included the following: 1) patients who had angina pectoris within the previous 6 months; 2) patients who had congestive heart failure and arrhythmia; 3) patients who had adverse cardiovascular and cerebrovascular events and coronary artery bypass grafting or an interventional operation within the previous 3 months; 4) patients who had taken opioids preoperatively; 5) patients who had a joint infection or systemic infection; 6) patients who had chronic pain caused by rheumatoid arthritis or bone tumors; and 7) patients who had other diseases or conditions that did not fit into the study after being assessed by the investigator. All patients were treated by the physicians of the same medical group. The patients were administered peripheral nerve blocks. During the surgery, the patients were administered anesthetics of the same category (a single-shot popliteal fossa nerve block; $20 \mathrm{~mL}$ of $0.25 \%$

Table I General demographic data of the patients in the three groups

\begin{tabular}{lllll}
\hline & Group F & Group C & Group BTDS & P-value \\
\hline Age (years) & $54.2 \pm 13.7$ & $53.1 \pm 17.6$ & $49.2 \pm 13.7$ & $\mathrm{~ns}$ \\
Gender, M/F & $3 / 27$ & $2 / 28$ & $3 / 27$ & $\mathrm{~ns}$ \\
Height $(\mathrm{cm})$ & $161.5 \pm 5.8$ & $163.7 \pm 6.1$ & $159.6 \pm 7.3$ & $\mathrm{~ns}$ \\
Weight $(\mathrm{kg})$ & $60.8 \pm 7.9$ & $58.7 \pm 6.9$ & $60.8 \pm 6.1$ & $\mathrm{~ns}$ \\
BMI $\left(\mathrm{kg} / \mathrm{m}^{2}\right)$ & $23.1 \pm 1.9$ & $22.0 \pm 1.7$ & $23.8 \pm 2.1$ & $\mathrm{~ns}$ \\
\hline
\end{tabular}

Notes: Group F: flurbiprofen axetil intravenous injection; Group C: oral celecoxib; and Group BTDS: buprenorphine transdermal delivery system. Data shown as mean \pm SD. ns: no significant difference between study groups.

Abbreviations: BTDS, buprenorphine transdermal delivery system; F, female; M, male; VAS, visual analog scale.

Table 2 Clinical data of the three groups of patients

\begin{tabular}{lllll}
\hline & Group F & Group C & Group BTDS & P-value \\
\hline VAS score before drug administration & $6.2 \pm 1.3$ & $6.2 \pm 1.1$ & $6.3 \pm 1.0$ & $\mathrm{~ns}$ \\
Preoperative hallux valgus angle $\left(^{\circ}\right)$ & $33.0 \pm 3.2$ & $34.1 \pm 2.8$ & $34.0 \pm 2.9$ & $\mathrm{~ns}$ \\
Preoperative intermetatarsal angle $\left({ }^{\circ}\right)$ & $14.0 \pm 2.2$ & $13.9 \pm 2.9$ & $14.1 \pm 1.7$ & $\mathrm{~ns}$ \\
Total surgical time (minutes) & $40.5 \pm 9.8$ & $40.8 \pm 9.7$ & $39.4 \pm 8.5$ & $\mathrm{~ns}$ \\
\hline
\end{tabular}

Notes: Group F: flurbiprofen axetil intravenous injection; Group C: oral celecoxib; and Group BTDS, buprenorphine transdermal delivery system. Data shown as mean \pm SD. ns: no significant difference between study groups.

Abbreviations: BTDS, buprenorphine transdermal delivery system; VAS, visual analog scale. 
preservative-free bupivacaine with 1:200,000 epinephrine). We conducted a distal soft tissue procedure ${ }^{12}$ combined with proximal chevron osteotomy ${ }^{13}$ and Akin osteotomy ${ }^{14}$ in all patients. A straight medial incision was routinely performed. The medial capsule was opened in a Y-shaped manner. The medial eminence was excised, and a normal V-type Chevron osteotomy was performed with a 5-6 $\mathrm{mm}$ lateral displacement of the distal fragment. In all patients, a $3.2 \mathrm{~mm}$ screw was used for the fixation of the metatarsal osteotomies and a $2.5 \mathrm{~mm}$ screw was used for the phalangeal fixation. Skin closure was performed with continuous subcuticular sutures using Vicryl 4-0. A surgical dressing was applied using a self-adherent wrap. Immediate weight bearing was allowed with a special postoperative shoe. Partial weight bearing was continued for $6-8$ weeks postoperatively.

\section{Medication regimen}

Group F: the patients were administered $50 \mathrm{mg}$ flurbiprofen axetil injection (Kaifen; Tide Pharmaceutical Co., Ltd, Beijing, People's Republic of China) through an intravenous injection twice per day beginning 2 days before the surgery, and the drug was continuously administered until the third day after the surgery. Group C: the patients received an oral administration of $200 \mathrm{mg}$ celecoxib twice per day beginning 2 days before the surgery, and the drug was continuously administered until the third day after the surgery. Group BTDS: the patients received the BTDS $(10 \mathrm{mg})$ on one-third of the lateral upper arm (right side or left side) 2 days before the surgery. If a skin defect or a large scar was detected on both upper arms, the BTDS could be attached to the upper chest. The patch was not removed during the surgery, and it was conserved until the fifth day after the surgery. Rescue analgesia was primarily provided by an intramuscular injection of $100 \mathrm{mg}$ tramadol hydrochloride when requested by the patient; we evaluate the "uncontrolled pain" with VAS value; if the average patient-report VAS $>6$ in the past 6 hours, rescue analgesia should be applied. If tramadol hydrochloride failed to relieve the pain within 60 minutes, then an intramuscular injection of $50 \mathrm{mg}$ pethidine was allowed.

\section{Observation indexes}

The degree of pain was evaluated using the VAS score: scores 0-2 indicated no pain; scores $3-5$ indicated mild pain; scores 6-8 indicated moderate pain; and scores 9-10 indicated severe pain. The VAS score of each patient was recorded at the following time points: on the night (at $8 \mathrm{pm}$ ) before surgery, at $8 \mathrm{am}$ on the first postoperative day, at 8 am on the second postoperative day, and at 8 am on the third postoperative day. The occurrence of adverse effects and the condition in which the patient was administered rescue analgesia for uncontrolled pain were also recorded. All patients were discharged at noon on the third postoperative day. Before discharge, the BTDS patients were given written instructions for removing the patch on the fifth postoperative day. In addition, all of the patients were asked to grade their satisfaction of the pain service as "good", "fair", and "unsatisfactory" before discharge, although they did not indicate the specific factors that inspired their feelings of satisfaction or dissatisfaction. The data collection was performed by a research assistant who was blinded to the subgrouping and administration of the procedures.

\section{Statistical analysis}

The statistical analysis was performed using SPSS Version 21.0 for Windows (IBM Corporation, Armonk, NY, USA). A statistician performed all statistical analyses. Continuous variables were expressed as the mean \pm standard deviation. Categorical data were presented as the counts and percentages. Student's $t$-test, the chi-square test, and Fisher's exact test were used to compare the two groups as appropriate. A one-way analysis of variance was applied to compare the data among different groups. A $P$-value of $<0.05$ was considered statistically significant.

\section{Results}

Patient disposition is shown in Figure 1. Of the 113 patients enrolled, 90 (eight males and 82 females) patients were randomized: 30 patients to the flurbiprofen axetil (intravenous) group, 30 patients to the celecoxib (oral) group, and 30 patients to the BTDS group. All of the patients in the three groups completed the study.

The VAS pain scores of the patients at each time point are presented in Table 3. All drug administrations in the three groups resulted in significant reduction in pain on the night before the surgery, the first postoperative day, the second postoperative day, and the third postoperative day. The VAS scores of the patients in Group F and Group BTDS were 2.4 and 2.5 , respectively, which were superior to those of the patients in Group C as 3.4 on the night before surgery. Similarly, the VAS scores of the patients in Group F (2.5) and Group BTDS (2.8) were lower than Group C (3.6) on the first postoperative day, $(P<0.001)$ as shown in Table 3 . However, no differences in the VAS scores of the three groups were observed on the second and third postoperative days $(P=0.44$ and 0.43 , respectively). In addition, significant differences 


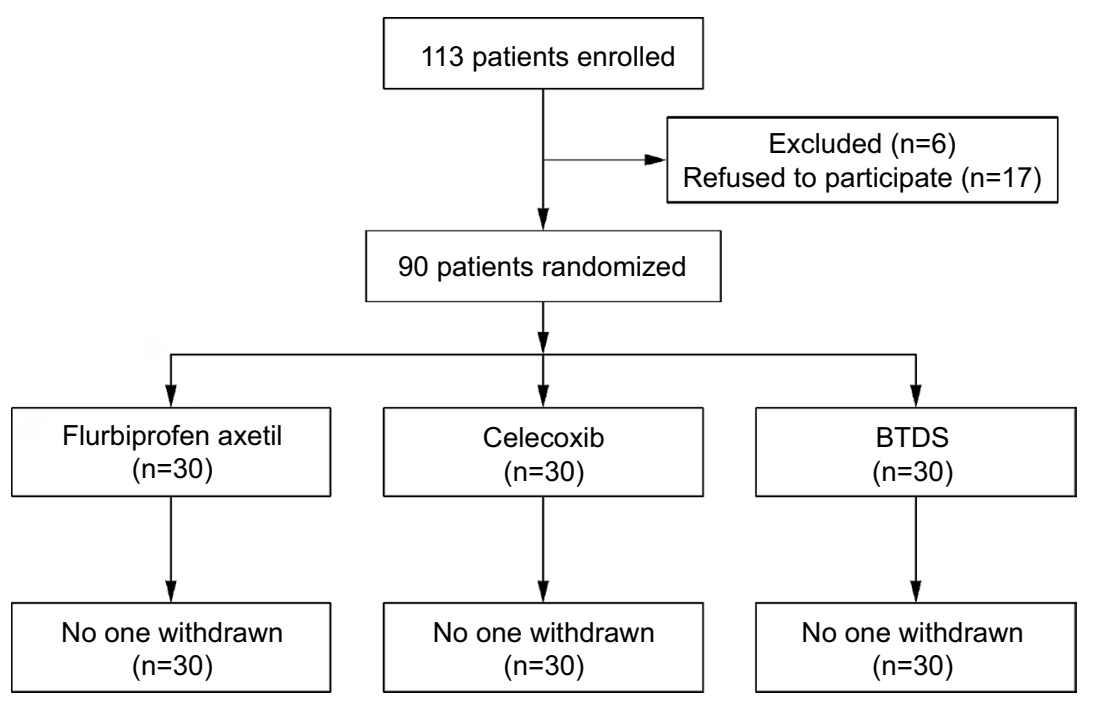

Figure I Patient disposition.

Abbreviation: BTDS, buprenorphine transdermal delivery system.

Table 3 Comparisons of the perioperative pain (VAS score) assessments between the three groups at different time points

\begin{tabular}{lllll}
\hline & Group F & Group C & Group BTDS & P-value \\
\hline Before drug administration & $6.2 \pm 1.3$ & $6.2 \pm 1.1$ & $6.3 \pm 1.0$ & $\mathrm{~ns}$ \\
Night before surgery & $2.4 \pm 1.0$ & $3.4 \pm 0.8$ & $2.5 \pm 0.9$ & $<0.00 I^{*}$ \\
Postoperative day I & $2.5 \pm 0.6$ & $3.6 \pm 0.5$ & $2.8 \pm 0.7$ & $<0.00 I^{*}$ \\
Postoperative day 2 & $1.8 \pm 0.7$ & $2.0 \pm 0.6$ & $1.9 \pm 0.5$ & $\mathrm{~ns}$ \\
Postoperative day 3 (first I2 hours) & $1.4 \pm 0.6$ & $1.7 \pm 0.7$ & $1.5 \pm 0.5$ & $\mathrm{~ns}$ \\
P-value & $<0.00 I^{* *}$ & $<0.00 I^{* *}$ & $<0.00 I^{* *}$ & - \\
\hline
\end{tabular}

Notes: Group F: flurbiprofen axetil intravenous injection; Group C: oral celecoxib; and Group BTDS, buprenorphine transdermal delivery system. *Significant differences between Group $C$ and Group F and between Group C and Group BTDS but no significant differences between Group F and Group BTDS. **Significant differences in the VAS scores of the groups on the night before surgery, postoperative day I, postoperative day 2, and postoperative day 3, when compared with the VAS scores before drug administration. Significant differences in the VAS scores between postoperative day I and postoperative days 2 and 3. Data shown as mean \pm SD. ns: no significant difference between study groups.

Abbreviations: BTDS, buprenorphine transdermal delivery system; VAS, visual analog scale.

were not observed in the VAS scores between Group F and Group BTDS at the aforementioned time points $(P>0.05)$.

A rescue injection of tramadol hydrochloride $(100 \mathrm{mg}$, intramuscular) was, respectively, administered in two, four, and one patient on the night of the surgery and five, seven, and four patients on the first postoperative day in Groups F, C, and BTDS (Table 4). The patients in our study had the worst pain and called for the rescue injection at 18 hours postoperatively, which may be related to the residual effect of the popliteal fossa nerve block on preventing pain during the earlier postoperative period. None of the patients required a further injection of pethidine. Statistically significant differences were not observed in the tramadol hydrochloride consumption between the three groups on the day of surgery and the first postoperative day. On the second postoperative day, the patients in Group F and Group BTDS did not need any rescue analgesic at all.

Differences were not observed between the number of patients suffering from nausea, constipation, dizziness, somnolence, vomiting, headache, and application site reactions (skin allergy and pruritus) between the groups (Table 5).
One patient in Group BTDS suffered from application site reactions, although this did not present significant differences when compared with the other two groups $(P>0.05)$. None of the patients in the three groups suffered from severe complications (eg, peptic ulcers, respiratory inhibition, and cardiovascular events).

The overall satisfaction of the pain service was significantly higher in Group BTDS than in Group F and Group C. Significant differences were not observed in the degree of patient satisfaction between Group F and Group C $(P>0.05)$. These results are listed in Table 6.

Suture reactions or mild superficial erythema resulting from a foreign body reaction to the suture material developed in the foot of two patients in Group F and in the foot of one patient in Group C. All of the feet healed with conservative management. No other wound complications or deep infections occurred.

\section{Discussion}

The gender proportion of hallux valgus deformity is 15 women for every one man (a 15:1 female:male ratio). ${ }^{15}$ 
Table 4 Summary of the use of rescue analgesia among the three groups

\begin{tabular}{lllll}
\hline & Group F $(\mathbf{n}=\mathbf{3 0})$ & Group C $(\mathbf{n}=\mathbf{3 0})$ & Group BTDS $(\mathbf{n}=\mathbf{3 0})$ & P-value \\
\hline Day of surgery & $2(6.6)$ & $4(13.3)$ & $\mathrm{I}(3.3)$ & $\mathrm{ns}$ \\
Postoperative day I & $5(16.6)$ & $7(23.3)$ & $4(13.3)$ & $\mathrm{ns}$ \\
Postoperative day 2 & $0(0)$ & $3(10)$ & $0(0)$ & $0.04 *$ \\
\hline
\end{tabular}

Notes: The values are expressed as the $\mathrm{n}(\%)$. Group F: flurbiprofen axetil intravenous injection; Group C: oral celecoxib; and Group BTDS, buprenorphine transdermal delivery system. *Significant differences were observed between Group $C$ and Group F and between Group $C$ and Group BTDS. ns: no significant difference between study groups.

Abbreviation: BTDS, buprenorphine transdermal delivery system.

Table 5 Comparison of the side effects of the three groups

\begin{tabular}{lllll}
\hline & Group F (n=30) & Group C (n=30) & Group BTDS (n=30) & P-value \\
\hline Nausea & $5(16.6)$ & $7(23.3)$ & $5(16.6)$ & $\mathrm{ns}$ \\
Constipation & $4(13.3)$ & $5(16.6)$ & $4(13.3)$ & $\mathrm{ns}$ \\
Dizziness & $4(13.3)$ & $3(10)$ & $3(10)$ & $\mathrm{ns}$ \\
Somnolence & $3(10)$ & $4(13.3)$ & $3(10)$ & $\mathrm{ns}$ \\
Vomiting & $2(6.6)$ & $\mathrm{I}(3.3)$ & $\mathrm{I}(3.3)$ & $\mathrm{ns}$ \\
Headache & $2(6.6)$ & $\mathrm{I}(3.3)$ & $\mathrm{I}(3.3)$ & $\mathrm{ns}$ \\
Application site reactions & 0 & 0 & $\mathrm{I}(3.3)$ & $\mathrm{ns}$ \\
\hline
\end{tabular}

Notes: The values are expressed as the $\mathrm{n}(\%)$. Group F: flurbiprofen axetil intravenous injection; Group C: oral celecoxib; and Group BTDS, buprenorphine transdermal delivery system. Application site reactions: skin allergy and pruritus. ns: no significant difference between study groups.

Abbreviation: BTDS, buprenorphine transdermal delivery system.

Table 6 Overall satisfaction of the three groups

\begin{tabular}{lllll}
\hline & Group F $(\mathbf{n}=\mathbf{3 0})$ & Group C $(\mathbf{n}=\mathbf{3 0})$ & Group BTDS $(\mathbf{n}=\mathbf{3 0})$ & P-value \\
\hline Good & II $(36.6)$ & $7(23.3)$ & $18(60)$ & $0.005^{*}$ \\
Fair & $9(30)$ & $11(36.6)$ & $6(20)$ & $\mathrm{ns}$ \\
Unsatisfactory & $5(16.6)$ & $5(16.6)$ & $4(13.3)$ & $\mathrm{ns}$ \\
Unknown & $4(13.3)$ & $6(20)$ & $5(16.6)$ & $\mathrm{ns}$ \\
\hline
\end{tabular}

Notes: The values are expressed as the $\mathrm{n}(\%)$. Group F: flurbiprofen axetil intravenous injection; Group C: oral celecoxib; and Group BTDS, buprenorphine transdermal delivery system. *Significant differences between Group $C$ and Group BTDS $(P=0.002)$ and between Group F and Group BTDS ( $P=0.03)$ but no significant differences between Group $F$ and Group $C(P=0.33)$. ns: no significant difference between study groups.

Abbreviation: BTDS, buprenorphine transdermal delivery system.

Unfortunately, the female patients seemed to be more sensitive to the adverse effects of strong pain medication, such as opioids, after forefoot surgery than the male patients. ${ }^{16}$ Therefore, multimodal analgesia is particularly important in the treatment of hallux valgus patients.

The use of multimodal analgesia could reduce postoperative pain, improve patient satisfaction, and decrease total opioid consumption in the postoperative period. A number of drug classes are currently being used in multimodal treatment strategies. NSAIDS, such as ibuprofen, indomethacin, and celecoxib, are commonly used in clinical practice; however, they can exert adverse effects of varying degrees on the digestive tract, platelets, and kidneys of patients. ${ }^{17}$ Acetaminophen is frequently used for the treatment of acute pain in the perioperative period because it can reduce the requirements for opioids and pain scores in patients. However, whether the intravenous administration of acetaminophen provides a significant benefit over the oral administration of acetaminophen remains unclear. ${ }^{18}$ Alpha-2 agonists have been shown to have opioid-sparing effects, although they can cause hypotension and bradycardia, which must be taken into consideration when administered. ${ }^{19}$ Preoperative dexamethasone, either given orally or given intravenously, has been found to reduce both the opioid consumption and the time to discharge; moreover, it reduces the incidence of nausea and is known to be an effective antiemetic. ${ }^{20}$

The BTDS is a type of synthetic opioid analgesic, and it was licensed for the treatment of moderate-to-severe chronic pain that does not respond to nonopioid analgesics. The active ingredient of the BTDS is buprenorphine, which is a potent opioid analgesic that acts primarily as a partial agonist at the $\mu$-opioid receptor. ${ }^{21}$ The BTDS offers many advantages over the typical full $\mu$-opioid receptor agonists for treating chronic pain, and its partial agonist activity induces a ceiling effect for respiratory depression but not for analgesia, thereby resulting in a reduced risk for this potentially fatal adverse event compared with that of other full opioid agonists. ${ }^{22}$

The BTDS is one option among multimodal analgesics, although postoperative pain is not an official indication for its use. This patch proceeds slowly, and $\sim 48$ hours are needed to 
reach the maximum buprenorphine concentrations after the initial patch application. ${ }^{23}$ Thus, the optimal time for placing the patch would be 2 days before surgery. In the present study, the patch was placed 2 days before the operation. Additionally, the intravenous injection of flurbiprofen axetil and oral administration of celecoxib were administered 2 days before surgery. Such regimens allowed for comparisons among these medications and also followed the principle of preemptive analgesia, which helps prevent the development of hypersensitivity in the perioperative period. ${ }^{24}$

Our study showed that the BTDS could effectively control perioperative pain in patients who underwent hallux valgus surgery. The analgesic effect of the BTDS was better than that of the oral administration of celecoxib. In addition, statistically significant differences were not observed in the VAS scores, adverse effects, and rescue analgesia use between the BTDS and the flurbiprofen axetil intravenous injection. However, the degree of patient satisfaction in the BTDS group was significantly higher $(P<0.05)$ compared with that of the other two groups because of the advantages of the approach used for the drug administration. Flurbiprofen axetil and celecoxib were administered twice per day, whereas the BTDS, which was administered on the local skin, could release a steady and continuous dose of buprenorphine over a period of up to 7 days, which confers the convenience of once-weekly dosing. This approach avoids oral and intravenous drugs and provides psychological comfort to the patients. ${ }^{25}$ Specifically for the patients presenting comorbid conditions and polypharmacy complications, the extended analgesia duration offered by the BTDS allowed less frequent dosing, which may help improve patient acceptability.

This study had certain limitations. The sample size in this study was limited; therefore, the findings cannot be applied to actual situations requiring the preemptive analgesia regimen of the BTDS on a large scale. Moreover, the following three groups in this study involved different modes of drug administration: intravenous, oral, and transdermal. Hence, a double-blind design could not be achieved and a bias from the experimental condition would be created. In addition, different pathways of drug administration might exert a certain degree of influence on the psychological statuses of the patients, which could result in errors in the study results. Therefore, large-sample double-blind controlled trials should be conducted in the future.

\section{Conclusion}

This study indicated that the BTDS (a preemptive analgesia regimen) could exert an analgesic effect during the perioperative period for patients who received hallux valgus surgery, which is important for sustaining postoperative physiological and psychological states and promoting functional rehabilitation.

\section{Acknowledgment}

This work was supported by the Mundipharma (China) Pharmaceutical Co., Ltd.

\section{Disclosure}

The authors report no conflicts of interest in this work.

\section{References}

1. Herr MJ, Keyarash AB, Muir JJ, Kile TA, Claridge RJ. Lateral transbiceps popliteal block for elective foot and ankle surgery performed after induction of general anesthesia. Foot Ankle Int. 2006;27(9):667-671.

2. Brattwall M, Turan I, Jakobsson J. Pain management after elective hallux valgus surgery: a prospective randomized double-blind study comparing etoricoxib and tramadol. Anesth Analg. 2010;111(2):544-549.

3. Kim BS, Shim DS, Lee JW, Han SH, Ko YK, Park EH. Comparison of multi-drug injection versus placebo after hallux valgus surgery. Foot Ankle Int. 2011;32(9):856-860.

4. Turan I, Assareh H, Rolf C, Jakobsson J. Multi-modal-analgesia for pain management after Hallux Valgus surgery: a prospective randomised study on the effect of ankle block. J Orthop Surg Res. 2007;2:26.

5. White PF, Issioui T, Skrivanek GD, Early JS, Wakefield C. The use of a continuous popliteal sciatic nerve block after surgery involving the foot and ankle: does it improve the quality of recovery? Anesth Analg. 2003;97(5):1303-1309.

6. Kapil RP, Cipriano A, Friedman K, et al. Once-weekly transdermal buprenorphine application results in sustained and consistent steadystate plasma levels. J Pain Symptom Manage. 2013;46(1):65-75.

7. Prausnitz MR, Langer R. Transdermal drug delivery. Nat Biotechnol. 2008;26(11):1261-1268.

8. Steiner D, Munera C, Hale M, Ripa S, Landau C. Efficacy and safety of buprenorphine transdermal system (BTDS) for chronic moderate to severe low back pain: a randomized, double-blind study. $J$ Pain. 2011;12(11):1163-1173.

9. Munera C, Drehobl M, Sessler NE, Landau C. A randomized, placebocontrolled, double-blinded, parallel-group, 5-week study of buprenorphine transdermal system in adults with osteoarthritis. J Opioid Manag. 2010;6(3):193-202.

10. Landau CJ, Carr WD, Razzetti AJ, Sessler NE, Munera C, Ripa SR. Buprenorphine transdermal delivery system in adults with persistent noncancer-related pain syndromes who require opioid therapy: a multicenter, 5-week run-in and randomized, double-blind maintenance-ofanalgesia study. Clin Ther. 2007;29(10):2179-2193.

11. Miller K, Yarlas A, Wen W, et al. Buprenorphine transdermal system and quality of life in opioid-experienced patients with chronic low back pain. Expert Opin Pharmacother. 2013;14(3):269-277.

12. Mann RA, Pfeffinger L. Hallux valgus repair. DuVries modified McBride procedure. Clin Orthop Relat Res. 1991;(272):213-218.

13. Sammarco GJ, Conti SF. Proximal Chevron metatarsal osteotomy: single incision technique. Foot Ankle. 1993;14(1):44-47.

14. Akin OF. The treatment of hallux valgus: a new operative procedure and its results. Med Sentinel. 1925;33:678-679.

15. Nery C, Coughlin MJ, Baumfeld D, Ballerini FJ, Kobata S. Hallux valgus in males - part 1: demographics, etiology, and comparative radiology. Foot Ankle Int. 2013;34(5):629-635.

16. Merivirta R, Pitkanen M, Alanen J, Haapoja E, Koivisto M, Kuusniemi K. Postoperative pain management with transdermal fentanyl after forefoot surgery: a randomized, placebo-controlled study. J Pain Res. 2015;8:39-45.

17. Bluhm B, Green LA. NSAID use associated with increased cardiovascular risk and death, but naproxen appears to be the least harmful. Evid Based Med. 2011;16(5):142-143. 
18. Raffa RB, Pergolizzi JV Jr, Taylor R Jr, Decker JF, Patrick JT. Acetaminophen (paracetamol) oral absorption and clinical influences. Pain Pract. 2014;14(7):668-677.

19. Ren C, Chi M, Zhang Y, Zhang Z, Qi F, Liu Z. Dexmedetomidine in postoperative analgesia in patients undergoing hysterectomy: a consort-prospective, randomized, controlled trial. Medicine (Baltimore). 2015;94(32):e1348.

20. Kim SY, Koo BN, Shin CS, Ban M, Han K, Kim MD. The effects of single-dose dexamethasone on inflammatory response and pain after uterine artery embolisation for symptomatic fibroids or adenomyosis: a randomised controlled study. BJOG. 2016;123(4):580-587.

21. Hoskin PJ, Hanks GW. Opioid agonist-antagonist drugs in acute and chronic pain states. Drugs. 1991;41(3):326-344.
22. Pergolizzi J, Aloisi AM, Dahan A, et al. Current knowledge of buprenorphine and its unique pharmacological profile. Pain Pract. 2010;10(5): 428-450.

23. Coplan PM, Sessler NE, Harikrishnan V, Singh R, Perkel C. Comparison of abuse, suspected suicidal intent, and fatalities related to the 7-day buprenorphine transdermal patch versus other opioid analgesics in the National Poison Data System. Postgrad Med. 2017;129(1):55-61.

24. Frerichs JA, Janis LR. Preemptive analgesia in foot and ankle surgery. Clin Podiatr Med Surg. 2003;20(2):237-256.

25. Tang J, Fan J, Yao Y, Cai W, Yin G, Zhou W. Application of a buprenorphine transdermal patch for the perioperative analgesia in patients who underwent simple lumbar discectomy. Medicine (Baltimore). 2017;96(20):e6844.
Journal of Pain Research

\section{Publish your work in this journal}

The Journal of Pain Research is an international, peer reviewed, open access, online journal that welcomes laboratory and clinical findings in the fields of pain research and the prevention and management of pain. Original research, reviews, symposium reports, hypothesis formation and commentaries are all considered for publication.

\section{Dovepress}

The manuscript management system is completely online and includes a very quick and fair peer-review system, which is all easy to use. Visit http://www.dovepress.com/testimonials.php to read real quotes from published authors. 